\title{
Strong-field molecular ionization: determination of ionization probabilities calibrated with field-free alignment.
}

\author{
Vincent Loriot, Edouard Hertz, Arnaud Rouzée, B. Sinardet, Bruno Lavorel, and Olivier Faucher \\ Laboratoire de Physique de l'Université de Bourgogne, UMR CNRS 5027, \\ B.P. 47870, 21078 Dijon Cedex, France
}

Compiled June 30, 2006

\begin{abstract}
We report an original optical method providing the probability of molecular ionization induced by femtosecond laser pulses. The approach consists of exploiting molecular alignment in order to extract reliable information about ionization. The cross defocusing technique implemented for this purpose reveals a sensitivity with respect to post-pulse alignment, as well as to free electron density induced by the ultra-short laser pulse. The analysis of the resulting signal gives thus access to absolute single-ionization probabilities calibrated through the degree of alignment provided that free electrons are mainly produced from single-ionization. The relevance of the method is assessed in $\mathrm{N}_{2}$. (C) 2006 Optical Society of America

OCIS codes: $190.7110,260.5210,300.6530$.
\end{abstract}

Interaction of molecules with intense ultrashort laser pulses has opened great experimental and theoretical fields of interest including for instance multiphoton ionization process, high-order harmonics generation, abovethreshold ionization, and Coulomb explosion. In this framework, a detailed knowledge of the fundamental single-ionization process is crucial since a wide array of phenomena is initiated from this precursory step. Substantial theoretical effort has been focused in this sense and some recent models dedicated to molecular strong field ionization have emerged. ${ }^{1,2}$ Another remarkable feature of nonperturbative molecular interaction concerns the possibility of manipulating molecules in space. It is now well established that molecules exposed to intense laser pulses can be aligned by the electric field. In case of non-resonant short pulses (compared to the rotational period), periodic molecular alignment takes place in field-free conditions at well defined times corresponding to the revival of the rotational wavepacket yield in the molecular ground state. ${ }^{3}$ The present work takes advantage of the coexistence of molecular alignment and ionization in order to provide absolute probabilities of single-ionization calibrated with post-pulse alignment. The applied method is based on the cross defocusing of a probe pulse which has demonstrated its relevance for determining the degree of alignment induced in $\mathrm{CO}_{2}$ with femtosecond pulses. ${ }^{4}$ The cross defocusing relies on the dependency of the refractive index upon alignment. A first "pump" pulse is focused in a molecular gas sample inducing post-pulse alignment. The degree of alignment, which depends on the laser intensity, is larger at the center of the laser focus than at the edge inducing thus a spatial gradient of the refractive index. This effect can be perceived as a time-dependent non-linear lens. A weak time-delayed "probe" pulse is then propagated in the medium. Depending on the delay, the latter will experience the index gradient and undergo modification of its divergence related to the degree of transient alignment. By detecting the modification of the probe size in the far field region as a function of the pump-probe delay, the field-free molecular alignment can be quantified as previously reported. ${ }^{4}$ When using high laser intensity, molecular ionization occurs during the interaction with the pump pulse. As explained in this paper, significant ionization induces an additional refractive index gradient that contributes therefore to the whole detected signal. The signal can thus provide access to ionization probabilities calibrated via the degree of field-free molecular alignment. Such approach for investigating strong field ionization should be considered in the context of techniques based on phase-sensitive measurement. ${ }^{5}$ The relevance of our method for the determination of ionization probabilities is demonstrated in nitrogen $\mathrm{N}_{2}$. The ionization probabilities extracted from experimental data are compared with values reported in litterature and found in excellent agreement.

The experimental arrangement has been described elsewhere. ${ }^{4}$ Briefly, a Ti:sapphire chirped pulse amplifier delivers pulses of $100 \mathrm{fs}$ duration around $800 \mathrm{~nm}$ at $1 \mathrm{KHz}$ repetition rate. The output beam is split in two parts to produce the pump and probe beams. The two beams are focused and crossed at small angle in a static cell filled with $\mathrm{N}_{2}$ at low pressure (between 0.1 and 1 bar). Pump and probe beams are linearly polarized along the same axis. The relative delay between them is adjusted via a motorized translation stage. The modification of the spatial profile of the probe beam is measured with a coronography-like technique. At the exit of the cell, a mask is used in order to hide the central part of the probe beam. The light passing around the mask is collected by a photomultiplier tube detector. The diameter of the mask is chosen to produce a signal close to zero for an isotropic molecular distribution in the cell. When molecular alignment occurs, a signal is detected as a result of the defocusing of the probe beam due to the spatial distribution of aligned molecules. In order to estimate accurately the peak intensity $I_{0}$ of the pump beam under which the experiment is conducted, its waist $\omega_{0}$ 
has been evaluated by recording the beam profile at the focus.

Theoretical analysis of alignment measured by cross defocusing technique has been detailed elsewhere ${ }^{4}$ and will be only briefly outlined below. The degree of alignment is quantified by the usual averaged value $\left\langle\cos ^{2} \theta\right\rangle$, where $\theta$ represents the angle between the molecular axis and the laser polarization direction. $\left\langle\cos ^{2} \theta\right\rangle>1 / 3$ corresponds to alignment (i.e. molecular axis preferentially aligned along the polarization direction) whereas $\left\langle\cos ^{2} \theta\right\rangle<1 / 3$ to planar delocalization (i.e. molecular axis preferentially delocalized into the plane perpendicular to the polarization direction). The alignment signal measured by cross defocusing technique is proportional to $\left(\left\langle\cos ^{2} \theta\right\rangle-1 / 3\right)^{2}$. It relies on the dependency of the refractive index upon molecular alignment. Along the laser polarization axis, the variation of the refractive index due to the time-delayed rotational molecular response is given by: $\Delta n_{r}(r, t) \simeq \rho \Delta \alpha /\left(2 \varepsilon_{0}\right) \times\left(\left\langle\cos ^{2} \theta\right\rangle(r, t)-1 / 3\right)$ where $r$ stands for the radial coordinate, $t$ the time, $\rho$ the molecular density, $\triangle \alpha$ the polarisability anisotropy $\left(8.24 \times 10^{-41} \mathrm{C} \mathrm{m}^{2} \mathrm{~V}^{-1}\right.$ for $\left.\mathrm{N}_{2}{ }^{6}\right)$, and $\varepsilon_{0}$ the vacuum permittivity. In $\mathrm{N}_{2}\left\langle\cos ^{2} \theta\right\rangle$ is approximately linear with respect to the pump intensity for intensities less than about $60 \mathrm{TW} / \mathrm{cm}^{2}$. As a result $\Delta n_{r}$, in regards to the prior equation, exhibits a spatial distribution identical to the pump beam. The laser profil at the focus is taken to be parabolic

$$
\triangle n_{r}(r, t)=\Delta n_{r_{0}}(t)\left(1-2 \frac{r^{2}}{\omega_{0}^{2}}\right)
$$

with $\triangle n_{r_{0}}(t)$ the variation of the refractive index at $r=0$. This assumption of parabolic profile allows to obtain a simple analytic solution for the signal expression. Following the procedure reported in Ref. 4, the cross defocusing signal detected for a probe pulse launched subsequently to the pump (so as to neglect the instantaneous electronic response) can be written:

$$
\begin{aligned}
S & \propto I_{p r}(t) \otimes \Delta n_{r_{0}}^{2}(t) \\
& \propto I_{p r}(t) \otimes\left(\left\langle\cos ^{2} \theta\right\rangle(r=0, t)-1 / 3\right)^{2},
\end{aligned}
$$

with $I_{p r}(t)$ the probe intensity. The signal is thus directly proportional to the degree of molecular alignment produced at the beam center $(r=0)$ with the peak intensity $I_{0}$. It does not arise from a standard spatial averaging which is actually intrinsic to the present model. For a pump pulse of high intensity, the ionization becomes significant. Under our experimental conditions, ionization leads to the creation of a plasma which manifests itself by a variation of the refractive index proportional to the plasma electron density $n_{e}: \triangle n=-n_{e} /\left(2 \times n_{c r}\right)<0$ $\left(n_{c r}=1.75 \times 10^{21} \mathrm{~cm}^{-3}\right.$ is the critical density at 800 $\mathrm{nm}){ }^{5}$ The refractive index depends on the electron density and therefore on the ionization probability. We will consider, in the intensity range of this work, a non-linear dependence of the last with respect to the pump intensity with a non-linearity factor $N_{L}$. Such assumption, generally possible in a limited range of intensity (as investigated in the present work), has been shown wellfounded ${ }^{8}$ in $\mathrm{N}_{2}$. The refractive index gradient associated to the plasma, considering the parabolic spatial profile of the pump aforementioned, can be then given by the following expression:

$$
\triangle n_{p}(r)=\triangle n_{p_{0}}\left(1-2 \frac{r^{2}}{\omega_{0}^{2}} N_{L}\right)
$$

where $\Delta n_{p_{0}} \propto \rho I_{0}^{N_{L}}$ is the variation at $r=0$ of the refractive index due to the plasma. In view of the pressure and delay range explored in this work, any relaxation phenomenon of plasma can be neglected so that the index gradient is assumed constant in time. The postpulse signal detected by the cross defocusing technique results now from the combination between alignment and plasma contributions. Eq.(2) can be thus modified as follow:

$$
\begin{aligned}
S & \propto I_{p r}(t) \otimes\left(\triangle n_{r_{0}}(t)+N_{L} \triangle n_{p_{0}}\right)^{2} \\
& \propto I_{p r}(t) \otimes\left(\left\langle\cos ^{2} \theta\right\rangle(r=0, t)-1 / 3+F\right)^{2}
\end{aligned}
$$

with $F=2 \varepsilon_{0} N_{L} \triangle n_{p_{0}} /(\rho \triangle \alpha)<0$ having through $\triangle n_{p_{0}}$ a non-linear dependence $N_{L}$ with respect to the peak intensity of the pump. According to the previous expression, ionization gives rise to an heterodyned signal leading to a modification of the transient shapes together with the apparition of a background. A set of scans at different pump intensities has been recorded and fitted using Eq.(4) with $F$ as a free parameter. Typical experimental and theoretical traces are depicted in Fig.1. The signal to noise ratio provided by the cross defocusing technique is high and the large background, mostly due to the plasma, appears clearly on the measured signal. The agreement between fitted and experimental traces is excellent and interestingly, the calculated trace by changing the $F$ parameter by $20 \%$ leads to significant disagreement particularly in terms of baseline as evidenced by the inset depicting the difference obs. - calc. for the two cases $(F$ and $0.8 \times F)$. This point suggests a notable sensitivity of the method with respect to ionization. The $F$ value extracted from fits is related to the electron density at $r=0$. In this work, single-ionization turns out to be the dominant process (compared to multiple ionization or dissociation) and the electron density thus found is assumed originating exclusively from single-ionization. ${ }^{7}$ Single-ionization probability at $r=0$ subject to the peak intensity $I_{0}$ is then given by:

$$
P\left(I_{0}\right)=\frac{n_{e}\left(I_{0}\right)}{\rho}=-\frac{2 n_{c r} \triangle n_{p_{0}}\left(I_{0}\right)}{\rho}=-\frac{n_{c r} \triangle \alpha F\left(I_{0}\right)}{\varepsilon_{0} N_{L}} .
$$

The $N_{L}$ parameter has been determined through the non-linear dependence of $F$ with respect to the peak intensity $I_{0}$. Our series of fits have revealed a value $N_{L}=7.5 \pm 0.5$ consistent with the value reported in Ref. 8 and the present ionization regime characterized 


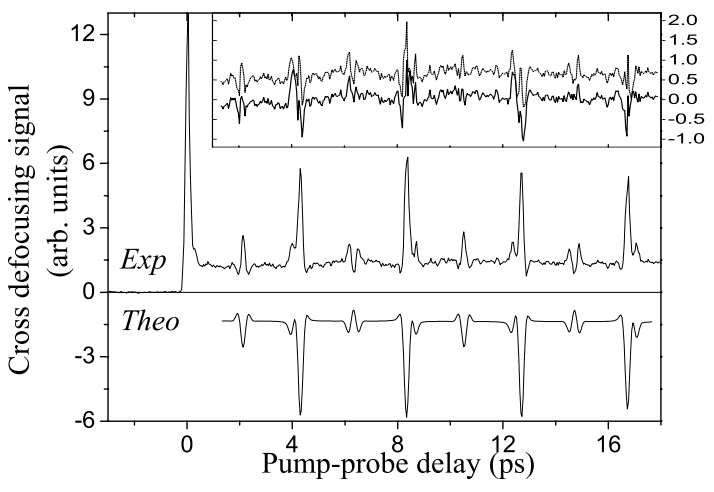

Fig. 1. Experimental trace and corresponding fit (inverted) in $\mathrm{N}_{2}$ with $I_{0}=58 \mathrm{TW} / \mathrm{cm}^{2}$. The inset displays obs. - calc. compared with the one (dashed) obtained by decreasing the $F$ factor by $20 \%$ (see text).

by a Keldish parameter $\gamma \approx 1$. The ionization probability versus intensity deduced from Eq.(5) is displayed in Fig. 2. Standard deviation associated to ionization probabilities in the range of intensity $\left[\approx 40-100 \mathrm{TW} / \mathrm{cm}^{2}\right]$ indicated by a shaded area on the figure lies within 1 and $10 \%$. At lower intensity, the signal is outweighted by the alignment contribution being thus detrimental to the precision of results. A modification of the experimental configuration using for instance crossed polarized pump and probe pulses could be implemented to balance the alignment response with the plasma contribution and extend the domain of reliability. At high intensity, the predominance of plasma in signal alters the accuracy of the results as well. Results are compared with

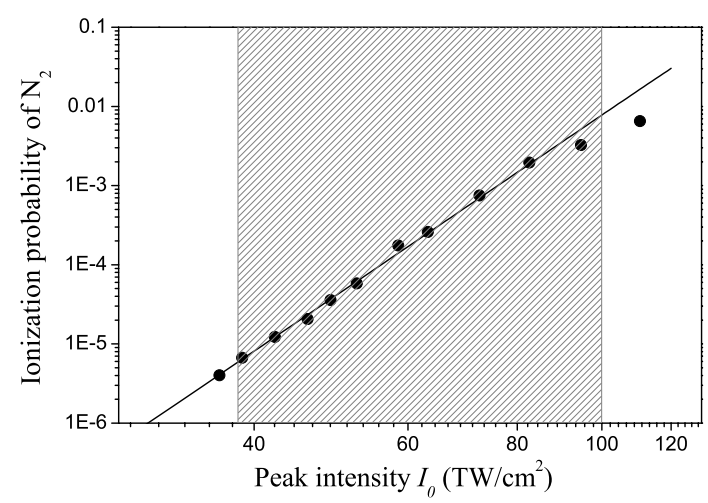

Fig. 2. (•) Ionization probability obtained from Eq.(5) compared with (full line) theoretical model (see text).

the semi-empirical model of Talepbour et al. ${ }^{9}$ relevant in the present regime. The ionization probabilities predicted by this model are calculated using the expression of the ionization rate given in Ref. 8 and plotted by multiplying the intensity by a factor only 1.2. As observed in Fig. 2, our experimental results conform to the model of Talepbour et al. below about $100 \mathrm{TW} / \mathrm{cm}^{2}$. Beyond this intensity, our technique predicts slightly smaller ionization than model. This discrepancy can possibly occurs because the initial assumption of $\left\langle\cos ^{2} \theta\right\rangle$ approximately linear with respect to the pump intensity fails. If so, we stress that a refined model with a more precise modelisation of the transverse profile of the index gradient should allow getting ride of this limitation. We argue nonetheless that the model of Talepbour suggests a drop in the slope of ionization around this intensity. This point is not included in the expression of the ionization rate of Ref. 8 used for the present calculation. The latter is indeed based on a power law dependance of ionization with respect to intensity that is valid only below $100 \mathrm{TW} / \mathrm{cm}^{2}$. To conclude, we emphasize that the cross defocusing method features several advantages to investigate laser-induced ionization. First, the ionization probability is determined in absolute by means of a simple experimental set-up without any inherent problems of calibration encountered with ion mass or photoelectron spectroscopy. Second, the ionization probability is determined at a given peak intensity without having to deal with spatial averaging. Third, the present method yields simultaneously monitoring of molecular alignment and ionization offering thus the opportunity for optimization of the alignment versus ionization by the use of pulse shaping technique. Finally, the method allows determination of the ionization ratio between molecules and their companion atoms by comparing traces of each species. The two last points will be investigated in a near future.

The authors acknowledge financial support by the Conseil Régional de Bourgogne, the CNRS, the ACI "Photonique", and a Marie Curie European Reintegration Grant within the 6th European Community RTD Framework Programme.

\section{References}

1. J. Muth-Böhm, A. Becker, F.H. M. Faisal, Phys. Rev. Lett. 85, 2280 (2000).

2. X. M. Tong, Z.X. Zhao, C. D. Lin, Phys. Rev. A 66, 0033402 (2002).

3. H. Stapelfeldt and T. Seideman, Rev. Mod. Phys. 75, 543 (2003).

4. V. Renard, O. Faucher, B. Lavorel, Opt. Lett. 30, 70 (2005).

5. C. W. Siders, G. Rodriguez, J. L. W. Siders, F. G. Omenetto, A. J. Taylor, Phys. Rev. Lett. 87, 263002 (2001).

6. M. A. Morrison, P. J. Hay, J. Chem. Phys. 70, 4034 (1979)

7. C. Cornaggia, Ph. Hering, Phys. Rev. A 62, 023403 (2000)

8. J. Kasparian, R. Sauerbrey, S. L. Chin, Appl. Phys. B $\mathbf{7 1}, 877$ (2000).

9. A. Talepbour, J. Yang, S. L. Chin, Opt. Comm. 163, 29 (1999). 